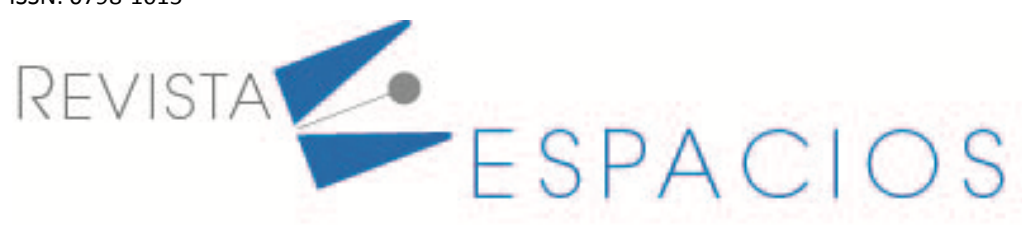

\title{
La educación inclusiva en el nivel inicial. Estudio de caso
}

\section{Inclusive education a principle of change at the initial level. Case study}

\author{
DELGADO, Karina E. ${ }^{1}$ \\ BARRIONUEVO, Lizeth. A. ${ }^{2}$ \\ ESSOMBA, Miquel A. ${ }^{3}$
}

\begin{abstract}
Resumen
El presente estudio analiza la educación de inclusión en el nivel de educación inicial, a través del Modelo Social de Educación Inclusiva que utiliza tres variables: i) políticas en educación inclusiva, ii) condiciones de los niños, y iii) actitudes asumidas hacia los niños. Se enmarca en una investigación cuantitativa de tipo ex post facto, aplicada en 30 centros infantiles, obteniéndose el índice de efectividad de la gestión en educación inclusiva de 51,3\%, relacionado con un bajo conocimiento para hacer inclusión a niños con discapacidad.

Palabras clave: educación inicial; inclusión; modelo e índice.
\end{abstract}

\begin{abstract}
The present study analyzes the education of inclusion at the level of initial education, through the Social Model of Inclusive Education which uses three variables: i) policies in inclusive education, ii) conditions of children, and iii ) assumed attitudes towards children. It is part of a quantitative ex post facto research, applied in 30 children's centers, obtaining the management effectiveness index in inclusive education of $51.3 \%$, related to a low knowledge to include children with disabilities.

Keywords: initial education; inclusion; model and index.
\end{abstract}

\section{Introducción}

La educación inclusiva en el nivel inicial se constituye en un principio de cambio, amparada según hechos en materia de una educación para todos, vista como un principio que aporta hacia la calidad educativa (Ainscow \& Booth, 2015). Se plantea a partir de tratados, convenciones, declaraciones y movimientos. Los hechos descritos se encaminan hacia un servicio educativo que busca cada vez ampliar su cobertura y lograr los aprendizajes de cada uno de los estudiantes en los niveles de educación inicial, media y superior.

\subsection{Movimientos para una educación inclusiva}

La Convención Internacional sobre protección de los derechos de todos los trabajadores migratorios y de sus familiares (1990), describe la situación de las personas en estado de migración y sus familias, por motivos de sexo, raza, color, idioma, religión o convicción, opinión política, o de otra índole. La Declaración de Salamanca

\footnotetext{
${ }^{1}$ Docente investigadora. Centro de de Investigación de Ciencias Humanas y de la Educación. Universidad Tecnológica Indoamerica. karinadelgado@uti.edu.ec

${ }^{2}$ Docente. Facultad de Ciencias Humanas y de la Educación. Universidad Tecnológica Indoamerica. lizethbarrionuevo@uti.edu.ec

${ }^{3}$ Docente. Departamento de Pedagogía Aplicada. Universidad Autónoma de Barcelona. MiquelAngel.Essomba@uab.cat
} 
(1994), desarrollada a través de la Conferencia Mundial sobre Necesidades Educativas Especiales, buscó promover el objetivo de una Educación para Todos, examinando los cambios fundamentales de políticas, necesarios para favorecer el enfoque de la educación integradora. El Foro Mundial de Educación para Todos de Dakar (UNESCO 2000), realizó un análisis respecto a los procesos de exclusión que continúan experimentando los grupos desaventajados, incluyendo a los niños, jóvenes y adultos trabajadores que viven en áreas rurales, que están afectados por conflictos, por VIH/SIDA, hambre, mala salud y aquellos con necesidades especiales de aprendizaje. La Convención Interamericana para la eliminación de todas las formas de discriminación contra las personas con discapacidad (Organización de Estados Americanos 2006), cuyos objetivos buscan prevenir y eliminar todas las formas de discriminación contra las personas con discapacidad y propiciar su plena integración en la sociedad. La Declaración Universal sobre la Diversidad Cultural (2002), ratifica que la cultura es un conjunto de rasgos distintivos, espirituales y materiales, intelectuales, y afectivos. Caracteriza a una sociedad o a sus diferentes agrupaciones, estos bienes serán preservados, valorados y transmitidos a las generaciones futuras. La Declaración de las Naciones Unidas sobre los Derechos de los Pueblos Indígenas (Agencia de las Naciones Unidas para los Refugiados 2006), describe los derechos a establecer y controlar sistemas, instituciones y docentes que impartan el servicio en sus propios idiomas, con sus métodos culturales de enseñanza y de aprendizaje. Los niños indígenas tienen derechos a todos los niveles y formas de educación. La Conferencia Internacional de Educación UNESCO celebrada en Ginebra (2008), analizó la relación entre la educación inclusiva, la sociedad y la democracia; el concepto de educación inclusiva y sus dimensiones operativas y, en particular, las principales características de las políticas y prácticas que pueden superar exitosamente la exclusión, tanto de la educación como a su interior, contribuyendo así a forjar sociedades más inclusivas, justas y equitativas. El Programa de las Naciones Unidas para el Desarrollo (Organización de las Naciones Unidas 2015), plantea trabajar con los Objetivos de Desarrollo Sostenible, que en materia de educación ocupa un lugar fundamental el objetivo 4, que describe "Garantizar una educación inclusiva, equitativa y de calidad y promover oportunidades de aprendizaje durante toda la vida para todos" (p.27). La Declaración de Lima, dada en una reunión ministerial, sobre Educación para todos en América Latina y el Caribe: Balance y desafíos post2015, buscó asegurar un acceso inclusivo y equitativo al aprendizaje de calidad para todos los niños, niñas, jóvenes y personas adultas, en todos los niveles del sistema educativo, desde la educación y el cuidado de la primera infancia.

Las razones descritas para una educación inclusiva, en el marco de tratados, convenciones, declaraciones y movimientos en el mundo, son un referente para el planteamiento de políticas educativas que abarcan a toda la diversidad de agrupaciones, descritas en el marco de una educación inclusiva, articuladas para el nivel inicial.

\subsection{Políticas de educación inclusiva}

Una sociedad equitativa implica el planteamiento y aplicación del derecho, descritos internacional y localmente para todos los sectores que la demandan (Meléndez, 2012). La educación inicial, como una oferta de estudios, debe hacer una descripción de las normativas que amparan un trabajo en educación inclusiva. La descripción se plantea según las Constituciones y Leyes, así como políticas de intervención, para la educación parvularia, pre escolar, inicial o pre básica. Es de gran importancia reconocer las legislaciones y su concreción a través de políticas públicas, institucionalizadas y con recursos suficientes para garantizar todas las especificidades de esta etapa, así como la existencia de mecanismos legales y judiciales que permitan la protección de los intereses, derechos y la reparación en casos de vulneración (Campaña Latinoamericana por el Derecho a la Educación Regional, Organización Mundial para la Educación Preescolar - Vicepresidencia Latina, 2018). La educación inclusiva en el nivel inicial, está fundamentada bajo principios como: la calidad, la equidad, la igualdad de oportunidades, el acceso sin distinciones ni privilegios y la atención a las necesidades educativas como elementos centrales del desarrollo (Parra, 2010). El nivel de educación inicial hace un reconocimiento a la inclusión, la prevención, la optimización de capacidades y la compensación de situaciones de desigualdad, dado que es una etapa excepcionalmente diversificadora por sus características que le son propias (Rodríguez, F. 2005). 


\subsection{Comprender a la educación inclusiva}

La educación inclusiva en este nivel se enfoca según las necesidades de atención considerando las particularidades de los niños que acceden. Se fundamenta en el constructivismo, al desafiar las prácticas pedagógicas tradicionales de la educación, asegura que los estudiantes tengan acceso a un aprendizaje significativo (Valenciano, 2009), desarrollado a partir de las conexiones entre lo nuevo y los esquemas previos que posee la persona. Las escuelas serán inclusivas, es decir, para todos, si los sistemas educativos aseguran que todos los alumnos tengan acceso a un aprendizaje significativo (Arnaiz, 2012).

Este estudio se enfoca hacia las necesidades de atención según las particularidades de los niños que acceden al nivel inicial. El nivel de educación inicial hace un reconocimiento a la inclusión, la prevención, la optimización de capacidades y la compensación de situaciones de desigualdad, (Rodríguez, F. 2005). El nivel inicial no aborda procesos de inclusión como tal, sin embargo, se ve la necesidad de valorar las acciones dadas en el marco de la educación inclusiva, con el fin a analizar su desarrollo. Por lo tanto, el objetivo es la detección temprana en caso de una condición de riesgo o, lo que es lo mismo, trabajar desde la prevención (Rodríguez et al., 2012). Para los autores Booth, Ainscow y Kingston (2007).

[...] tiene que ver tanto con la participación de los profesionales, como con la implicación de los niños. Participar implica jugar, aprender y trabajar en colaboración con otros. Implica hacer elecciones y opinar acerca de lo que estamos haciendo. En último término, tiene que ver con ser reconocido, aceptado y valorado por uno mismo (p.3).

Las descripciones dadas por los autores, se han agrupados según las diferentes condiciones de los estudiantes como condiciones sociales descritas por Cano (2017) y Guzmán et al., (2019), interculturales citadas por Cano (2017) y Guzmán et al., (2019) y/o condiciones personales definidas por Cano (2017) y Dosil \& Fuentes (2014). Las condiciones sociales e interculturales las fusionan los autores Cabrera, Calle y Cabrera (2019). Las descripciones, se ratifican desde los marcos normativos y según diferentes contextos. El Cuadro 1 categoriza la atención a la diversidad, permite orientar de mejor manera la terminología utilizada para hacer una educación inclusiva en el nivel inicial.

Cuadro 1

Categorización para atención a la diversidad de niños del nivel inicial

\begin{tabular}{|l|c|l|}
\hline \multicolumn{1}{|c|}{ Condición social } & Condición intercultural & \multicolumn{1}{c|}{ Condición personal } \\
\hline Lugar o situación de origen & Cultural & Discapacidad \\
Situación de riesgo & Diversidad & Retraso madurativo en el \\
Religión e ideología & Identidad & desarrollo \\
Pasado judicial de los padres & & Precocidad intelectual \\
Situación socio-económica & & \\
Emergencias sanitarias & & \\
\hline
\end{tabular}

Fuente: Autoras

Las categorías antes descritas se pueden presentar desde el nacimiento del niño, y su interacción con el mundo. La interacción podrá ser evidenciada por conductas o estatus marital maternos o paternos (Vargas \& Martínez, 2019), o por retrasos en hitos normales en cada etapa de vida. Las intervenciones se definirán de acuerdo con las necesidades individuales de cada niño y paulatinamente se ajustarán según la frecuencia, intensidad y duración se someterá a ellos un sujeto en función de su progreso (Rodríguez et al., 2012) basada en la estimulación.

Los argumentos planteados para hacer una educación inclusiva orientan con un nuevo reto de trabajo, debido a que es una etapa excepcionalmente diversificadora por las características que le son propias de la edad. La 
educación inclusiva en este nivel atiende las condiciones sociales, interculturales y/o personales de los niños. La inclusión trabaja con la estimulación como estrategia de prevención, optimización de capacidades y compensación de situaciones de desigualdad, busca la participación de los niños, la familia y profesionales, a través del juego, el aprendizaje y el trabajo entre todos.

\subsection{Un modelo de atención}

El modelo social de educación inclusiva: actitudes hacia los niños (MSEI), orienta la gestión en educación inclusiva de una manera integral. El MSEl se ha diseñado por los autores, según un análisis realizado con otros modelos como: i) Modelo inglés de indicadores de educación inclusiva, cuya aplicación se ha dado en todos los niveles educativos. En el nivel inicial, evalúa cómo se están llevando a cabo las prácticas inclusivas, qué culturas se sustentan y en qué medida pueden modificarse las políticas organizativas para ser más incluyentes, así como una transformación a nivel social, al implicar a toda la comunidad educativa y trascender al entorno local (Booth, Tony. \& Ainscow, 1998). Sin embargo, es necesario ampliar a mayor profundidad en las dimensiones de cultura y prácticas inclusivas, así como modificar el procedimiento de presentación de la herramienta para su juicio. ii) Modelo español para la evaluación de prácticas educativas del profesorado: Indicadores de mejora desde la educación inclusiva, diseñado para valorar las prácticas educativas del profesorado, analiza la manera en que éstas inciden de forma directa en la organización y planificación del trabajo de aula, considera las diferencias que presentan los estudiantes respecto a deficiencias visuales, deficiencias auditivas, deficiencias físicas, trastornos del lenguaje, deficiencias psíquicas, dificultades de aprendizaje, provenientes de situaciones sociales desfavorecidas, sobredotación intelectual y otras causas (Lledó y Arnaiz, 2010). Lo que muestra que hay únicamente un análisis relacionado con las condiciones personales asociadas a una discapacidad. iii) Modelo británico de evaluación respecto a resultados esperados en los aprendizajes de los estudiantes, muestra de manera específica cómo funciona la co-responsabilidad, dado mediante una gestión relacionada con el seguimiento y evaluación de desempeño y producción de resultados de aprendizaje. Los modelos tienen una escasa estrategia integral de atención a la diversidad o su relación con la educación inclusiva, han manejado procesos aislados que, de alguna manera, dan respuesta a cierta población de niños o atienden dimensiones que deberán ampliar su incidencia.

El desarrollo de un modelo social, aborda una atención basada en las limitaciones que genera la misma sociedad, los servicios apropiados y una adecuada atención educativa. Lo que significa que no se niega los problemas existentes dentro de una sociedad, sino se los debe situar como parte de ella. Por lo citado y partiendo de la premisa de que toda vida es igualmente digna, desde un modelo social se sostiene que el aporte hacia la diversidad de las personas, se encuentra íntimamente relacionado con la inclusión y la aceptación de las diferencias que pueden evidenciar, (Palacios, 2008).

Un modelo social, como parte de una sociedad democrática es una alternativa de trabajo que busca construir procesos educativos encaminados a atender necesidades que cada vez busca ser más equitativa. El MSEI establece un vínculo sostenido y de diálogo con las propias sociedades, que se encuentran en responsabilidad con la educación inicial, (Organización de las Naciones Unidas para la Educación la Ciencia y la Cultura, 2018).

\section{Metodología}

Este artículo describe los principales resultados desarrollados en el marco de una investigación cuantitativa, de tipo ex post facto o no experimental, siendo un estudio que no tiene ningún control, relacionado con las variables independientes. La metodología se desarrolla mediante un estudio correlacional que busca realizar un diagnóstico respecto a la educación inclusiva, para conocer en qué medida los Centros Infantiles (Cl), a través de las autoridades, educadores y niños aplican las políticas dispuestas respecto a la educación inclusiva, según las 
diferentes condiciones que presentan los niños, atendidas según la actitud que asuman las autoridades y educadores de los $\mathrm{Cl}$, constituyéndose en variables que caracterizan al MSEI. El Gráfico 1, permite visualizar lo descrito.

\section{Gráfico 1}

Relación de variables que integran el Modelo Social de Educación Inclusiva

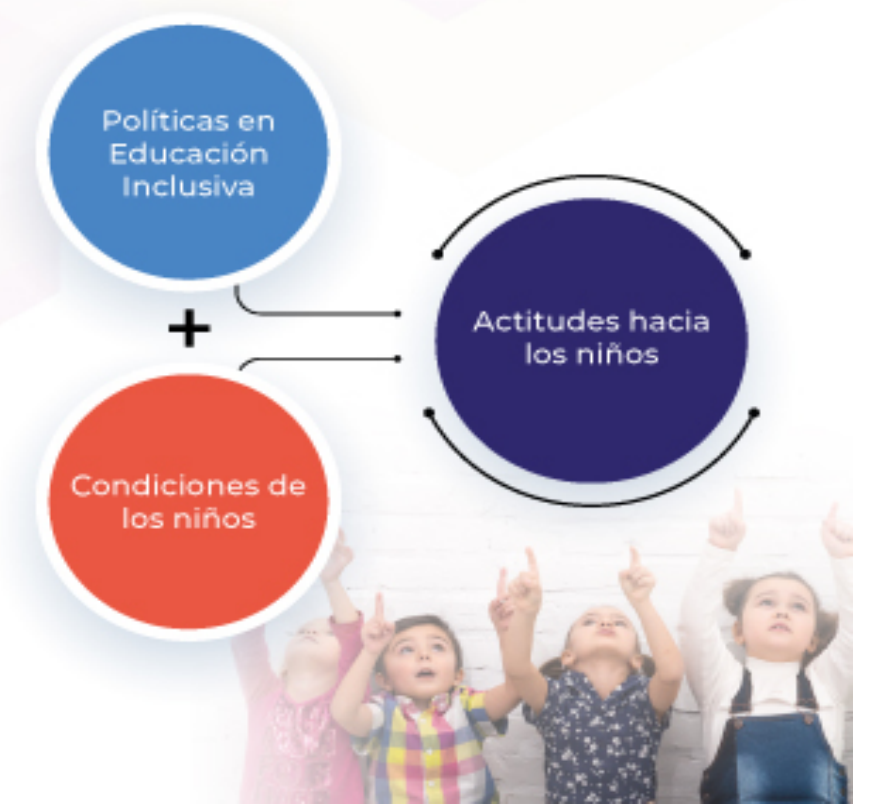

Fuente: (Delgado, 2019)

EI MSEI, requiere considerar realidades donde los elementos no son intercambiables entre sí, sino que mantienen una dependencia recíproca y adquieren sentido en función de un todo, (Gairín, 2015). La descripción operativa de las variables se detalla según: i) variables independientes: políticas en educación inclusiva (PEI), planteada según las normativas vigentes para generar un trabajo inclusivo desde cada uno de los actores, así como los fundamentos para hacer una educación inclusiva, y condiciones de los niños (CN), considera lo social, lo intercultural y/o personales, planteadas según las diferencias que puedan presentar los niños, descritas en las Tabla 1, y ii) variable dependiente: actitudes hacia los niños (AHN), planteada según las diferentes formas de abordar las condiciones que pueda presentar un niño, siendo un trabajo realizado para lograr el desarrollo de destrezas en los niños.

EI MSEI se analiza a través del modelo de ecuaciones estructurales o causales (MEE), permite establecer las relaciones de dependencia entre las variables, basado en el cálculo del índice de efectividad de la gestión en educación inclusiva, dado en una institución del nivel inicial o en una agrupación de estas, según criterios de conveniencia. Las variables utilizadas para el MEE los constituyen las PEI y las CN, así como AHN. Los MEE pueden ser expresados por medio de diagramas estructurales en los cuales las flechas indican la dirección de la influencia de una variable sobre la otra, tomando en cuenta las variables consideradas en el modelo de estudio se muestra el Gráfico 2. 
Gráfico 2

Diagrama estructural del Modelo de Ecuaciones Estructurales

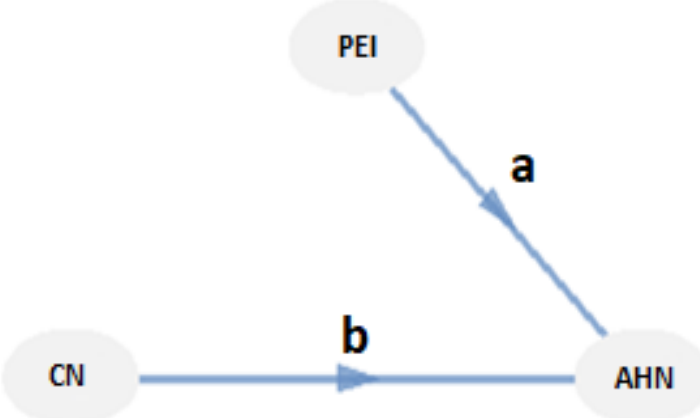

Fuente: Autores

EI MSEI describe como variable dependiente a la AHE, por tanto, desarrolla una sola ecuación estructural, según $\mathrm{AHN}$ es una función aditiva de los valores de las variables PEI y $\mathrm{CN}$, cuya fórmula es: $\mathrm{AHN}=\mathrm{a} * \mathrm{PEI}+\mathrm{b} * \mathrm{CN}$. La ecuación estructural de este modelo, permite calcular los valores a y b que se denominan pesos o coeficientes de las variables, estos valores son indicadores de la influencia sobre la variable AHN. La ecuación permite calcular el índice de efectividad de la gestión en educación inclusiva para las instituciones del nivel inicial, de forma equivalente como se calcula el índice de satisfacción en los modelos ECSI (European Customer Satisfaction Index) (Sánchez, 2013). Con el fin de aplicar esta metodología, el ajuste del modelo propuesto se realiza usando el paquete plspm del software estadístico R, utiliza mínimos cuadrados parciales como técnica estadística de ajuste. El parámetro que utiliza el paquete plspm mide el desempeño del modelo denominado GoF (Goodness of Fit).

Las variables utilizadas en el MSEI, desarrollan dimensiones y categorías, descritas en el Cuadro 2.

Cuadro 2

Descripción de variables, indicadores y categorías del MSEI

\begin{tabular}{|c|c|c|}
\hline Variables & Dimensiones & Categorías \\
\hline & Marco normativo. & $\begin{array}{l}\text { Políticas educativas en educación inclusiva (PEI 1). } \\
\text { Trabajo interministerial garantiza el desarrollo infantil integral (PEI 2). } \\
\text { Trabajo interministerial aplica el desarrollo infantil integral (PEI 3). } \\
\text { Protocolo para realizar apoyos o adaptaciones temporales o permanentes } \\
\text { (PEI 4). } \\
\text { Apoyos o adaptaciones temporales o permanentes para atender a los } \\
\text { niños (PEI 5). } \\
\text { Documentos curriculares que garanticen el trabajo en educación inclusiva } \\
\text { (PEI 6). }\end{array}$ \\
\hline $\begin{array}{l}\text { V1: Políticas en } \\
\text { educación } \\
\text { inclusiva (PEI). }\end{array}$ & $\begin{array}{l}\text { Instructivo de } \\
\text { regulación y } \\
\text { estándares de } \\
\text { aplicación. }\end{array}$ & $\begin{array}{l}\text { Proyecto Educativo Institucional en el nivel (PEI 7). } \\
\text { Proyecto Educativo Institucional refleja una cultura institucional } \\
\text { integradora, inclusiva y flexible (PEI 8). } \\
\text { Proyecto Educativo Institucional construido en comunidad (PEI 9). } \\
\text { Autoevaluación en el Centro Infantil (PEI 10). } \\
\text { Planes de mejora institucionales como parte del Proyecto Educativo } \\
\text { Institucional (PEI 11). } \\
\text { Plan de mejora institucional aplicado (PEI 12). } \\
\text { Ingreso de los niños con un reporte médico (PEI 13). } \\
\text { Protocolos de atención en caso de afecciones de los niños (procesos) (PEI } \\
\text { 14). }\end{array}$ \\
\hline
\end{tabular}




\begin{tabular}{|c|c|c|}
\hline Variables & Dimensiones & Categorías \\
\hline & $\begin{array}{l}\text { Descripción del } \\
\text { concepto de } \\
\text { educación inclusiva. }\end{array}$ & $\begin{array}{l}\text { Educación inclusiva aborda un trabajo de atención a todos los niños (PEI } \\
\text { 15). } \\
\text { Educación inclusiva considera condiciones sociales, interculturales y } \\
\text { personales de los niños (PEI 16). } \\
\text { Protocolos integrales de atención, en casos de educación inclusiva (PEI } \\
\text { 17). } \\
\text { Casos de atención relacionados con la educación inclusiva (PEI 18). }\end{array}$ \\
\hline \multirow{3}{*}{$\begin{array}{l}\text { V2: Condiciones } \\
\text { de los niños (CN). }\end{array}$} & Sociales. & $\begin{array}{l}\text { EL CI niega el ingreso de algún niño y niña por alguna situación específica } \\
\text { (PEI 19). } \\
\text { En el Cl cuántos niños son considerados como casos de vulnerabilidad } \\
\text { (madres adolescentes, padres vendedores ambulantes, familia con } \\
\text { problemas con la ley, niños a cargo de hermanos mayores, violencia } \\
\text { intrafamiliar) (PEI 20). }\end{array}$ \\
\hline & Interculturales. & $\begin{array}{l}\text { El Cl niega el ingreso de algún niño y niña por alguna condición } \\
\text { relacionada con su cultura, diversidad o identidad (PEI 21). } \\
\text { El Cl cuanta con niños indígenas (PEI 22). } \\
\text { El Cl cuenta con niños afroecuatorianos (PEI 23). } \\
\text { El Cl cuneta con niños de otras nacionalidades (PEI 24). }\end{array}$ \\
\hline & Personales. & $\begin{array}{l}\text { El Cl cuanta con niños con alguna condición de discapacidad (PEI 25). } \\
\text { El Cl cuanta con niños con un desarrollo evolutivo no acorde a la edad } \\
\text { (PEI 26). } \\
\text { El Cl cuanta con niños con un desarrollo fisiológico no acorde a la edad } \\
\text { (PEI 27). } \\
\text { Cantidad de niños que cumplen con las destrezas acordes a su edad (PEI } \\
28) .\end{array}$ \\
\hline \multirow{3}{*}{$\begin{array}{l}\text { V3: Actitudes } \\
\text { hacia los niños } \\
\text { (AHN). }\end{array}$} & $\begin{array}{l}\text { Principios básicos del } \\
\mathrm{Cl} \text { en función de una } \\
\text { atención equitativa. }\end{array}$ & $\begin{array}{l}\text { Casos de atención por algún tipo de discapacidad (PEI 29). } \\
\text { El Cl atiende diferentes necesidades en los niños (PEI 30). }\end{array}$ \\
\hline & $\begin{array}{l}\text { Equipo responsable } \\
\text { del } \\
\text { acompañamiento. }\end{array}$ & $\begin{array}{l}\text { Actividades de actualización para mejorar su desempeño laboral (PEI 31). } \\
\text { Apoyos para jornadas de actualización para mejorar su desempeño } \\
\text { laboral (PEI 32). } \\
\text { Participa procesos de capacitación relacionados con la inclusión (PEI 33). } \\
\text { Apoyos de profesionales especialistas, según alguna necesidad en los } \\
\text { niños (PEI 34). } \\
\text { Niveles de instrucción (PEI 35). }\end{array}$ \\
\hline & $\begin{array}{l}\text { Procesos de la } \\
\text { institución para hacer } \\
\text { una educación } \\
\text { inclusiva. }\end{array}$ & $\begin{array}{l}\text { Interacción del } \mathrm{Cl} \text { con Centro Salud del sector (PEI 36). } \\
\mathrm{Cl} \text { cuenta con espacios físicos adecuados para infantes con discapacidad } \\
\text { física (PEI 37). } \\
\text { Documentos que maneja el CI para garantizar un proceso inclusivo (PEI } \\
\text { 38). } \\
\text { Estrategias aplicadas para niños que no se ha logrado alcanzar las } \\
\text { destrezas (PEI 39). }\end{array}$ \\
\hline
\end{tabular}

Fuente: Autores

Las categorías permiten desarrollar ítemes, se estructuran en instrumentos como el guión de entrevista, ficha de observación y el cuestionario. Los instrumentos sirven para recoger datos que proporcionan los encuestados en un ambiente de relación directa con los informantes, a través de un conjunto de preguntas o cuestiones. Los instrumentos son medidos con escala de Likert con un rango de 1 a 3, sinedo 1 en desacuerdo (ED), 2 medianamente de acuerdo (MA) y 3 totalmente de acuerdo (TA), así como una opción de respuesta en caso de no aplicar (NA) el indicador. 
EI MSEI, como un plan piloto se aplicó en Ecuador, en 30 Centros de Desarrollo Infantil (CDI) pertenecientes al Distrito Metropolitano de Quito. Los CDI son financiados por el Ministerio de Inclusión Económica y Social. Los CDI están representados por coordinadoras y educadoras, así como los padres de familia, su atención se centra en el nivel inicial I destinado para niños de 0 a 3 años de edad.

\section{Resultados}

\subsection{Población de estudio}

Los 30 Centros de Desarrollo Infantil de Ecuador, son de sostenimiento estatal o fiscal, sectorizados en igual proporción al norte, centro y sur del Distrito, ubicados en sectores marginales. La Figura 1 muestra la población de niños atendidos.

Figura 1

Población de niños de 30 CDI del Distrito Metropolitano de Quito

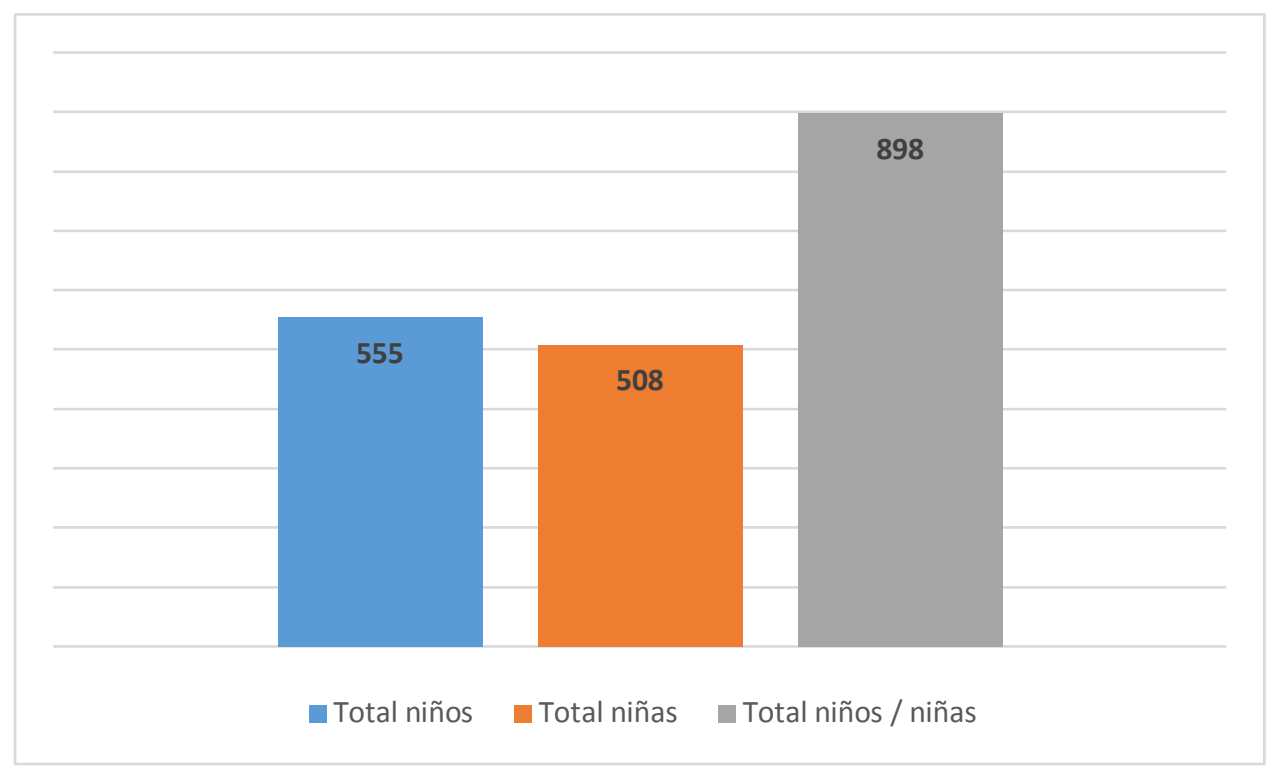

Fuente: Autores

La población de niños perteneciente a $30 \mathrm{CDI}$, evidencia una proporción un tanto equitativa en cuanto al género de los niños. El total de niños se constituye en una población bastante grande respecto a la atención en la primera infancia, considerándose que se hace efectivo el derecho a una atención del servicio educativo en los primeros niveles de estudio, pese a la gran demanda que tienen, lo que recae en que los niños que no logran ingresar, se acogen a otras modalidades de atención, ofertas desarrolladas por la municipalidad, acuden a $\mathrm{Cl}$ privados o no ingresan al nivel inicial. Si se realiza una valoración respecto a la atención por cada uno de los sectores como norte, centro y sur del Distrito Metropolitano de Quito, se evidencia la Figura 2. 
Figura 2

Población de niños por estratos en los 30 CDI del Distrito Metropolitano de Quito

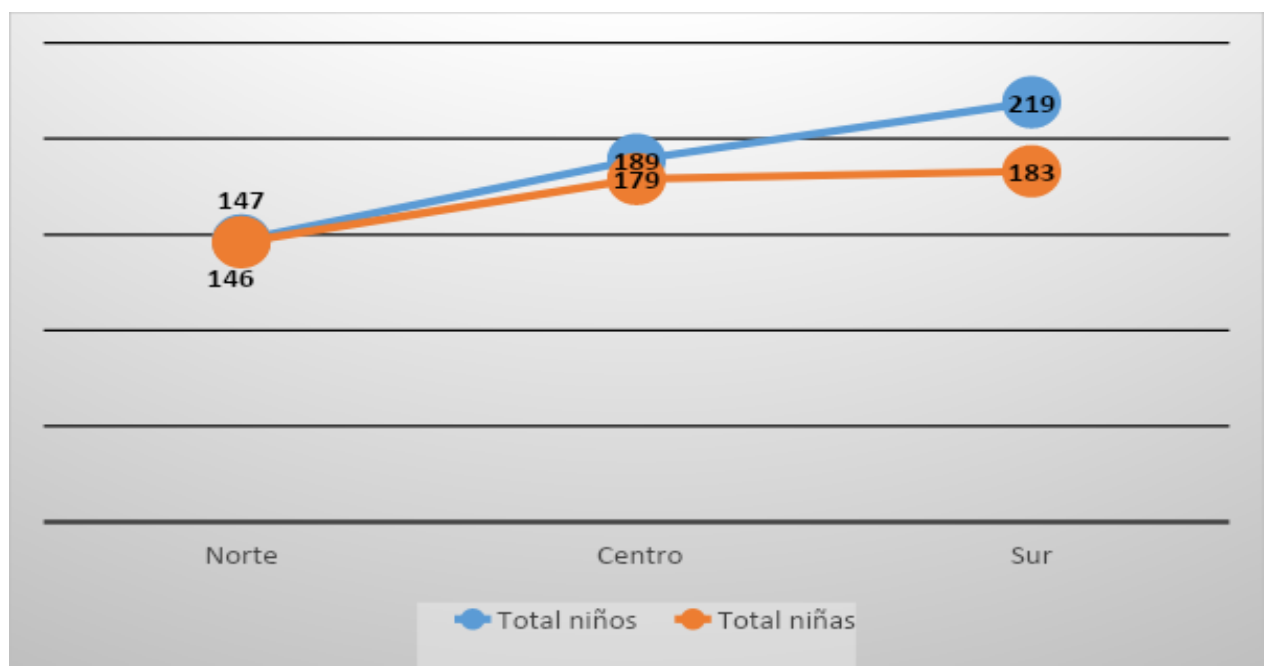

Fuente: Autores

La población por estratos, evidencia una mayor cantidad de niños en el sur del Distrito, siendo un sector con mayor población y de menores ingresos en ciertos sectores. La figura ratifica cierta equidad de género respecto a ingresos de niños y niñas en el nivel inicial I en los tres estratos analizados. El estudio se realizó con la participación de toda la comunidad educativa: coordinadoras de los CDI (26), educadoras (135), y padres de familia y/o representantes (33). Las coordinadoras y educadoras casi en la totalidad son de sexo femenino.

EI MSEI aplicado como un plan piloto realiza una revisión profunda de los aportes de todas las encuestadas, según las variables y categorías y sus correspondientes ítemes.

\subsection{Variable independiente: Políticas en educación inclusiva}

La variable latente políticas en educación inclusiva (PEI), agrupa como dimensiones al marco normativo, instructivo de regulación y estándares de aplicación, y la descripción del concepto de educación inclusiva aplicada para el nivel inicial. A manera de ejemplo se hará un detalle del análisis de la primera dimensión sobre el marco normativo, con el fin de generar un proceso de análisis respecto al desarrollo de cada una de las dimensiones, evidenciadas en las Figuras 3 y 4.

Figuras 3 y 4

Categorías relacionadas con la dimensión marco normativo
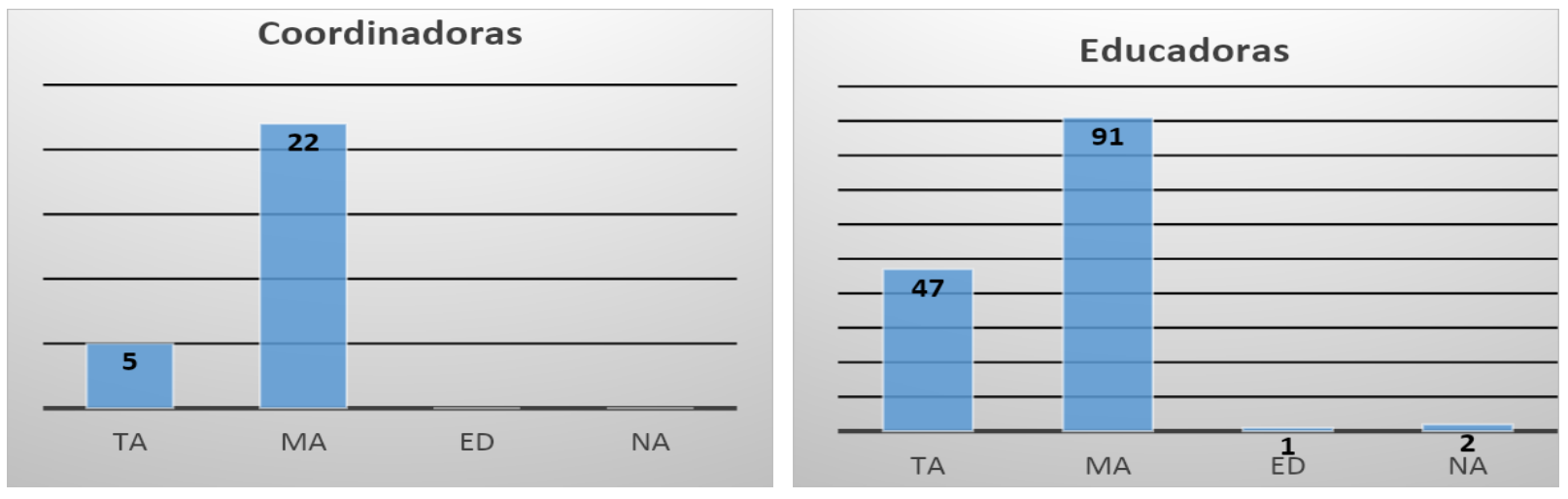

Fuente: Autores 
Las políticas dadas en el marco de la educación inclusiva, así como las políticas públicas de desarrollo infantil, son aplicadas medianamente por las coordinadoras y educadoras de los CDI. Los resultados reflejan que las coordinadoras han puntuado con menores pesos a las variables como las políticas educativas en inclusión (PEI 1) y la falta de protocolo para realizar apoyos o adaptaciones temporales o permanentes para los niños (PEI 4). Mientras que las educadoras han puntuado con menores pesos a las variables indicadoras como las políticas educativas en educación inclusiva (PEI 1) y la existencia de documentos curriculares que garanticen el trabajo en educación inclusiva (PEI 6). La variable indicadora políticas en educación inclusiva (PEI 1) genera una coincidencia respecto a los pesos en las respuestas tanto en coordinadoras como educadoras, reflejando la necesidad de operativizar las normas para lograr el trabajo en cada uno de los CDI.

Por otro lado, se muestra las Figuras 5 y 6, que totalizan la variable PEI.

Figuras 5 y 6

Variable latente PEI y sus variables indicadoras
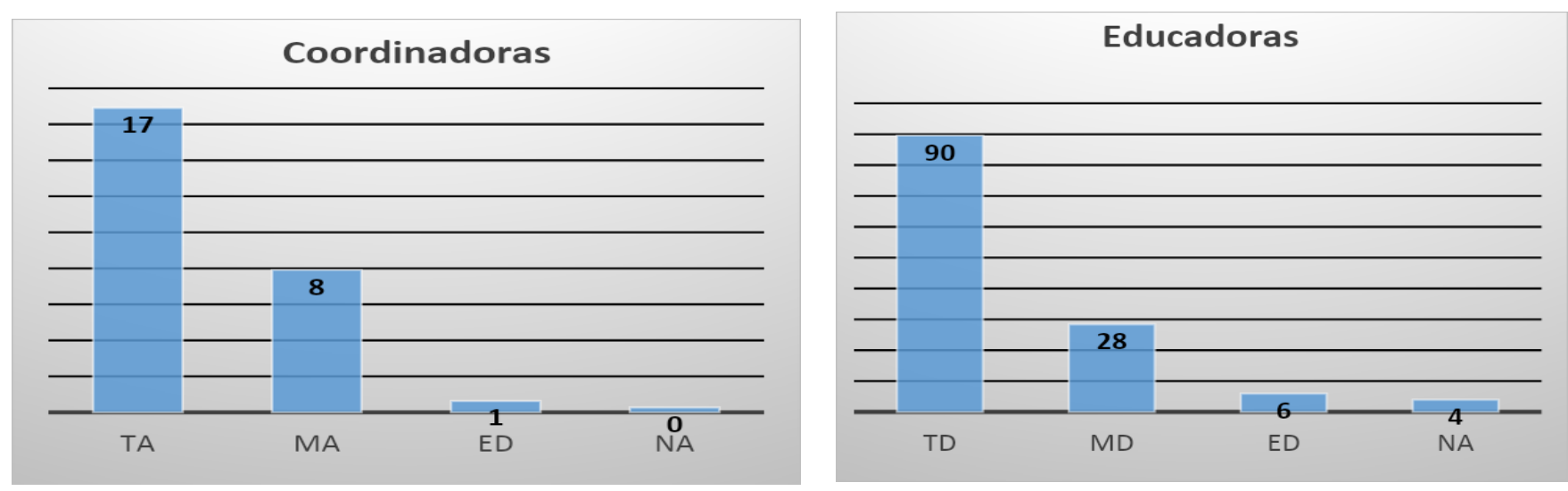

Fuente: Autores

La variable PEI resulta del trabajo con las tres dimensiones, y sus correspondientes categorías, según lo detalla la Tabla 2, siendo las de mayor peso para las educadoras: la existencia de protocolos para realizar apoyos o adaptaciones temporales o permanentes (PEI 4) y la existencia de documentos curriculares que garantizan el trabajo en educación inclusiva (PEI 6). La categoría PEI 6, es ratificada por las coordinadoras, detallando la existencia de un proyecto educativo institucional para cada uno de los CDI, que aborda a la educación inclusiva y garantiza una autoevaluación de los centros y la generación de planes de mejora. Las coordinadoras son las responsables de generar el mencionado instrumento curricular. Sin embargo, se describe las categorías de menor peso que demandarán acciones de intervención respecto a las políticas educativas en educación inclusiva (PEI 1) y la necesidad de documentos curriculares que garanticen el trabajo en educación inclusiva (PEI 6).

\subsection{Variable independiente: Condiciones de los niños}

La variable condiciones de los niños (CN) agrupa como dimensiones a las condiciones sociales, interculturales y/o personales de los niños, así como sus correspondientes categorías descritas en el Cuadro 2. Las Figuras 7 y 8 totalizan a las categorías. 
Figuras 7 y 8

Variable latente $\mathrm{CN}$ y sus variables indicadoras
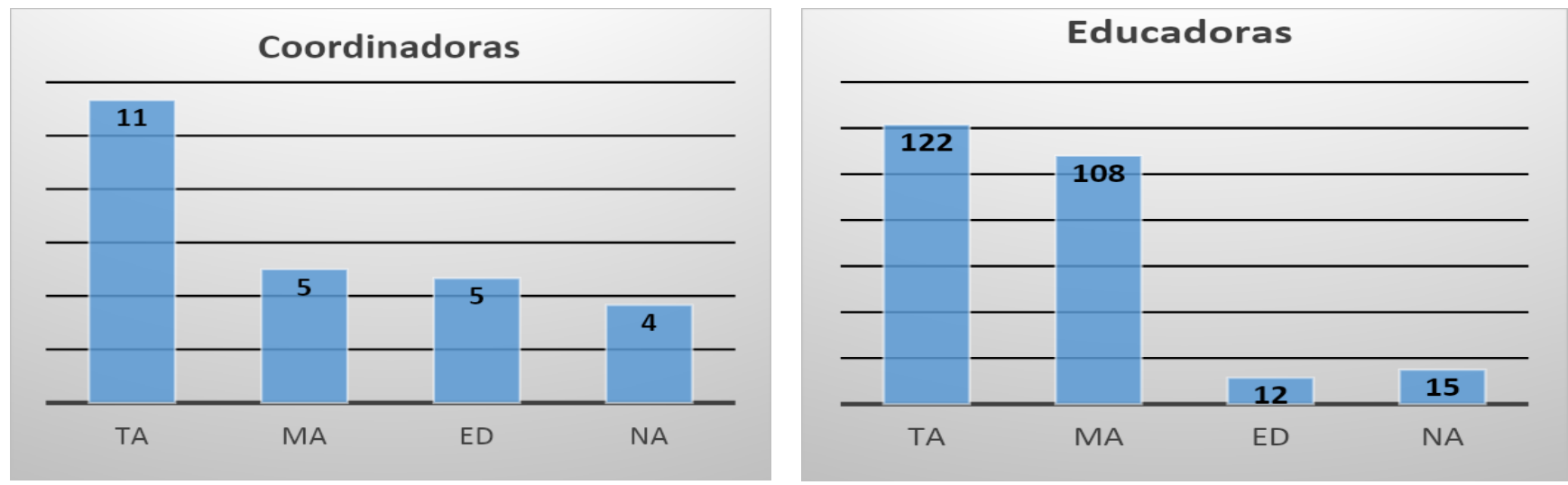

Fuente: Autores

La variable latente $\mathrm{CN}$, resulta de la relación con tres dimensiones, y sus correspondientes categorías, siendo las de mayor peso para las coordinadoras los ítemes que indagan sobre el ingreso a los CDI de niños con alguna condición de discapacidad (PEI 25), mientras que para las educadoras las categorías de mayor peso constituyen los ítemes relacionados con las categorías que indagan sobre el ingreso a los CDI de niños con alguna condición de discapacidad (PEI 25), niños con un desarrollo evolutivo no acorde a la edad (PEI 26) y con un desarrollo fisiológico no acorde a la edad (PEI 27). Los CDI son instituciones totalmente inclusivas, en relación con las dimensiones de condiciones sociales e interculturales de los niños, ya que un referente de atención de estos centros es atender a población infantil de familias de bajos recursos económicos o en situación de vulnerabilidad.

\subsection{Variable dependiente: Actitudes hacia los niños}

La variable actitudes hacia los niños (AHN), agrupa como dimensiones: principios básicos del CDI en función de una atención equitativa, equipos responsables del acompañamiento y procesos de la institución para hacer una educación inclusiva. Las Figuras 9 y 10 totalizan las categorías que son parte de AHN.

Figuras 9 y 10

Variable latente $\mathrm{AHN}$ y sus variables indicadoras
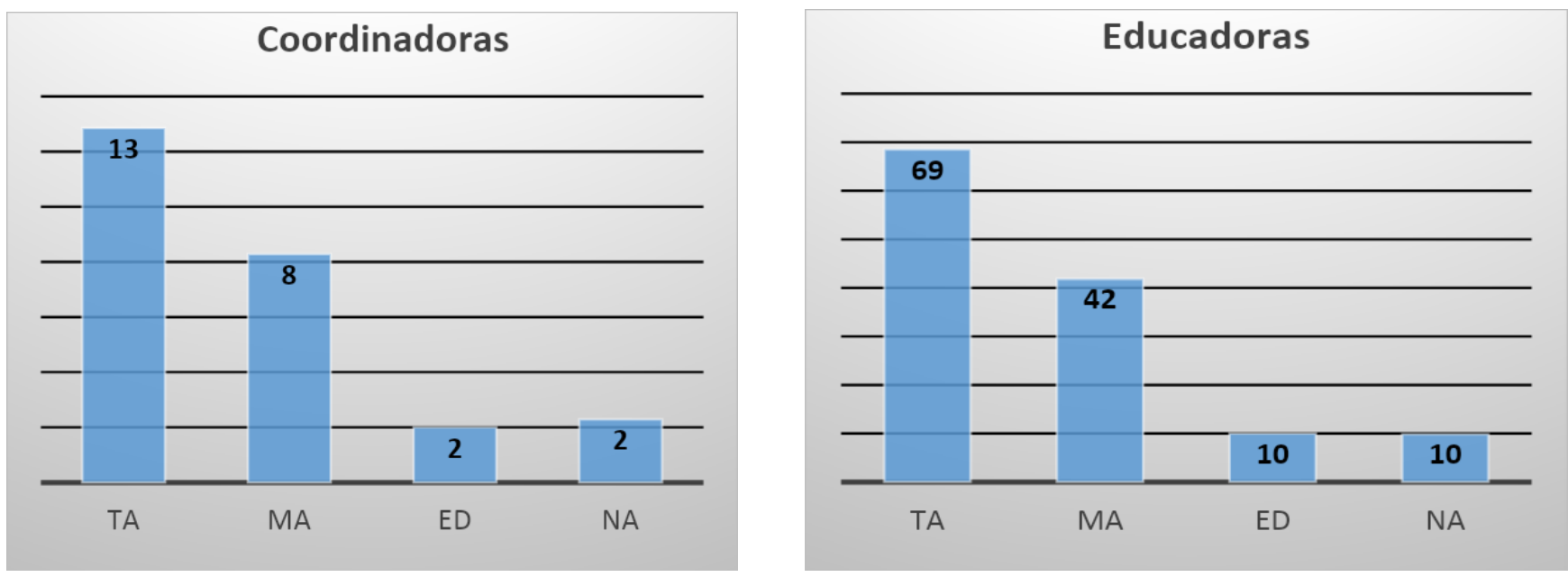

Fuente: Autores

La variable latente AHN está formada por tres dimensiones y sus correspondientes categorías, siendo las de mayor peso para las coordinadoras: las actividades de actualización para mejorar su desempeño laboral (PEI 31), 
los apoyos para jornadas de actualización para mejorar su desempeño laboral (PEI 32), la participación en procesos de capacitación relacionados con la inclusión (PEI 33) y los apoyos de profesionales especialistas, según alguna necesidad en los niños (PEI 34). Mientras que las educadoras, referencian como categorías de mayor peso a: las actividades de actualización para mejorar su desempeño laboral (PEI 31) y la interacción del Cl con el Centro Salud del sector (PEI 36).

Por otro lado, se analiza que la mayoría del personal encuestado manifiesta participar de actividades para su desempeño laboral, sin embargo, mencionan la necesario de recibir capacitaciones en temas de inclusión. Las educadoras y algunas coordinadoras desconocen los diferentes tipos de estrategias que deben emplear en los casos de inclusión. Es de gran importancia citar que algunas educadoras están en procesos de titulación en su formación inicial, demandan de una mejor preparación para la atención, educación y protección de los niños.

\section{Conclusiones}

Respecto a los hallazgos en este estudio que analiza a la educación inclusiva en el nivel inicial, basada en un principio de cambio, con la aplicación del "Modelo social de educación inclusiva”, se muestran:

La educación inclusiva en el nivel inicial debe comprenderse como un nuevo reto de trabajo, debido a que es una etapa excepcionalmente diversificada por las características que le son propias de la edad; atiende las condiciones sociales, interculturales y/o personales de los niños. La inclusión trabaja con la estimulación como estrategia de prevención, optimización de capacidades y compensación de situaciones de desigualdad, busca la participación de los niños, la familia y los docentes, a través del juego, el aprendizaje y el trabajo entre todos. Lo descrito permite comprender que la inclusión se concibe como un proceso de cambio en el que se debe iniciar.

En relación con el MSEl, primeramente, es necesario describir que el éxito o fracaso de la gestión en educación inclusiva en el nivel inicial resulta de la influencia de factores, desde el macrosistema (normas en el marco de la educación inclusiva), el exosistema (entornos a los que pertenecen los niños) y el microsistema (familias y centro infantil) (Formichella \& Kruger, 2019). Lo que genera una relación con las variables como políticas en educación inclusiva, condiciones de los niños y actitudes hacia los niños planteadas respectivamente con el planteamiento del modelo.

EI MSEl propone tres variables de gran importancia PEI, CN y AHN, así como una métrica basada en un índice de efectividad. El modelo va más allá de los modelos tradicionales que evalúan de una manera aislada a la inclusión o que, en el nivel inicial no han sido desarrollados. Si bien la necesidad es extensiva, este tipo de modelo ofrece la oportunidad de indagar de una forma más holística y multidimensional a la gestión de la educación inclusiva. EI MSEI utiliza el MEE ajustado con mínimos cuadrados parciales, siendo una metodología para estimar el nivel de efectividad de la gestión en educación inclusiva, así como su desempeño. Es necesario tener en cuenta que un modelo podrá tener un buen ajuste con una muestra, esto no significa que puedan existir otros modelos que también se ajustan muy bien a los datos muéstrales, en ese sentido siempre será interesante poder contrastar otros modelos que también sean sustentados con la teoría propuesta en este estudio o por otras teorías alternativas.

En relación con el pilotaje del MSEI, aplicado en una zona del territorio ecuatoriano en $30 \mathrm{CDI}$ estatales, permitió su validación, reflejando una relación entre las variables. Al aplicarse el MEE se muestra el índice de efectividad de la gestión en educación inclusiva, siendo 51,3\%, relacionado con un trabajo de mayor peso en categorías como: la existencia de protocolos para hacer una educación inclusiva en relación con las diferentes condiciones de los niños; la existencia de un proyecto educativo con acciones que respalden la gestión en educación inclusiva, que englobe una cultura inclusiva; la atención a niños con desarrollo evolutivo tardío; la interacción con centros de salud del sector; la atención de los CDI prioriza la alimentación, atención médica, seguida del aprendizaje, 
aseo y descanso. Lo señalado refleja los indicadores con mayor aceptación de cumplimiento en relación con la gestión en educación inclusiva. De la misma manera el MSEI permite conocer las categorías de menor peso, y tomar decisiones relacionadas con medidas de apoyo e intervención. El MSEl también evidenció su desempeño, con un porcentaje de 87,6 siendo un modelo con una aceptabilidad en su aplicación.

Por último, cabe destacar que algunos de los hallazgos expuestos dan lugar a nuevas líneas de investigación. Particularmente a partir de las variables y dimensiones descritas que actúan como modeladoras del MSEI, lo que permitirá derivar categorías según otros contextos locales e internacionales en $\mathrm{Cl}$.

\section{Referencias bibliográficas}

Agencia de las Naciones Unidas para los Refugiados (2006). Declaración de las Naciones Unidas sobre los derechos de los pueblos indígenas. https://www.un.org/esa/socdev/unpfii/documents/DRIPS_es.pdf

Ainscow, M. y Booth, T. (2015). Guía para la Educación Inclusiva Desarrollando el aprendizaje y la participación (Grafilia (ed.); 1er ed.).

Arnaiz, P. (2012). Escuelas eficaces e inclusivas: cómo favorecer su desarrollo Effective and inclusive schools: How to promote their development. Educatio Siglo XXI, 30(1), 25-44.

Booth, T. y Ainscow, M. (Eds). (1998). From them to us: An international study of inclusion in education. London: Routledge. 1-21.

Booth, T., Ainscow, M. y Kingston, D. (2007). Index para la Inclusión: Desarrollo del juego, el aprendizaje y la participación en la Educación Infantil. España: CSIE

Booth, T. y Ainscow, M. (2015). Guía para la Educación Inclusiva. Desarrollando el aprendizaje y la participación en los centros escolares. Madrid: Grafilia.

Cabrera, J. M., Calle, J. P. y Cabrera, E.L. (2019). La inclusión en el aula en escuelas con alto rendimiento escolar: Estudio de caso en escules de la provincia de Carchi, Ecuador. Revista Espacios. 40 (44), 3. Recuperado de: https://www.revistaespacios.com/a19v40n44/a19v40n44p03.pdf

Campaña Latinoamericana por el Derecho a la Educación - Regional, Organización Mundial para la Educación Preescolar - Vicepresidencia para América Latina. (2018). El derecho a la educación y al cuidado en la primera infancia: Perspectivas desde América Latina y El Caribe.

Cano, M., Buenstado, M., Gutierrez, P., López, M. y Naranjo, A. (2017). ¿Qué es una Universidad Corporativa? Vértice. https://www.vertice.org/blog/una-universidad-corporativa/

Delgado, K. (2019). La educación inclusiva en América Latina: Una cuestión de actitud. https://amzn.to/2MT700Y

Delgado, K. (2019). El desafío de la educación inclusiva. Plan piloto en Ecuador. Universidad Tecnológica Indoamérica.

Dosil, F. y Fuentes, M. (2014). DIVERSIDAD CULTURAL, INTERCULTURALIDAD Y EDUCACIÓN INDÍGENA EN MEXICO. 19, 13-19. file://C:/Users/Matías Valencia/Downloads/Dialnet-

DiversidadCulturalInterculturalidadYEducacionIndig-5833853.pdf 
Formichella, M. M. y Krüger, N. (2019) "Condiciones de educabilidad y resultados escolares en barrios vulnerables de la ciudad de Bahía Blanca, Argentina". OBETS. Revista de Ciencias Sociales, 14(1): 89-118. doi: 10.14198/OBETS2019.14.1.03

Gairín, J. (2015). Los sistemas de acceso, normativa de permanencia y estrategias de tutoría y retención de estudiantes en educación superior (S. A. Wolters Kluwer España (ed.)).

Guzmán, C., Quezada, A., Durán, M. y Caro, A. (2019). La diversidad cultural en la universidad Autónoma de Nayarit. 1, 71-78. file://C:/Users/Matías Valencia/Downloads/795-3058-1-PB.pdf

Lledó, A. y Arnaiz, P. (2010). Asunción Lledó Carreres y Pilar Arnaiz Sánchez Revista Iberoamericana sobre Calidad, Eficacia y Cambio en Educación. Red de Revistas Científicas de América Latina, El Caribe, España y Portugal, 8, 96-109. http://www.rinace.net/reice/numeros/arts/vol8num5/art6.pdf\%0A

Meléndez, F. (2012). Instrumento internacional sobre derechos humanos aplicables a la administración de justicia. Estudio constitucional comparado. (8), 21-33.

Naciones Unidas. Derechos Humanos (1990). Convención internacional sobre la protección de los derechos de todos los trabajadores migratorios y de sus familiares. Adoptada por la Asamblea General en su resolución $45 / 158$.

Organización de Estados Americanos (2006). Convención Interamericana para la eliminación de todas las formas de discriminación contra las personas con discapacidad.

https://www.oas.org/juridico/spanish/tratados/a-65.html

Organización de las Naciones Unidas para la Educación la Ciencia y la Cultura. La Educación Superior (2018). http://www.siteal.iipe.unesco.org/sites/default/assets/pdf/eje/siteal_educacion_superior_201808.pdf

Organización de las Naciones Unidas: “Informe 2015 sobre los Objetivos de Desarrollo del Milenio”, Nueva York, 2015. [edición electrónica] https://repositorio.cepal.org/bitstream/handle/11362/40155/24/S1801141_es.pdf

Educación inclusiva: Un modelo de educación para todos. ISEES: Inclusión Social y Equidad En La Educación Superior, 8, 73-84.

Palacios, A. (2008). El modelo social de discapacidad: orígenes, caracterización y plasmación en la Convención Internacional sobre los Derechos de las Personas con Discapacidad. Madrid, España: CINCA.

Rodríguez, F. (2005). Bases Pedagógicas de la atención temprana: la atención a la primera infancia desde u contexto educativo; diseño curricular de la educación infantil. La escuela infantil.

Rodríguez, M., Moreno, A. y Axpe, M. (2012). La acción inclusiva para la mejora de habilidades de lenguaje oral y de lectura inicial en niños con Trastorno Específico del Lenguaje (TEL). Revista de Educacion, 359, 332356. https://doi.org/10.4438/1988-592X-RE-2011-359-097

Sánchez, G. (2013). PLS Path Modeling with R Trowchez Editions. Berkeley.

Urton, K., Wilbert, J. y Hennemann, T. (2015). Teachers' Attitude Towards Inclusion and Their Self - Efficacy. Psychologie in erziehing und unterricht, 62 (2), 147 -157.

UNESCO (Ed.) (1994). Declaración de Salamanca y Marco de Acción sobre Necesidades Educativas Especiales. Conferencia Mundial sobre Necesidades Educativas Especiales: Acceso y calidad. Salamanca, España: Autor. 
UNESCO (2000). Foro Mundial sobre la Educación Dakar, Senegal.

http://iin.oea.org/Cursos_a_distancia/Lectura\%2017_disc.Dakar.pdf

UNESCO (2002). Declaración Universal sobre la Diversidad Cultural. UNESCO. https://n9.cl/o30nx

UNESCO (Ed.) (2008). Conferencia Internacional de Educación. Cuadragésima octava reunión. UNESCO. https://n9.cl/3nye

Valenciano, G. (2009). Construyendo un Concepto de Educación Inclusiva: Una experiencia compartida. Revista Aspectos clave de la Educación Inclusiva, 13 - 24.

Vargas, E. y Martínez, G. (2019) "The association between early childhood education of 3-5 year olds and maternal marital status in Mexico". OBETS. Revista de Ciencias Sociales, 14(2): 529-550. doi:

10.14198/OBETS2019.14.2.09

Esta obra está bajo una Licencia Creative Commons Attribución-NoCommercial 4.0 International

(cc) BY-NC 